\title{
Estratégias adotadas para a garantia dos direitos da pessoa com câncer no âmbito Sistema Único de Saúde (SUS)
}

\author{
Strategies adopted to ensure the rights of people with cancer \\ in the Brazilian Unified Health System (SUS)
}

Mario Jorge Sobreira da Silva (https://orcid.org/0000-0002-0477-8595) ${ }^{1}$

Claudia Garcia Serpa Osorio-de-Castro (http://orcid.org/0000-0003-4875-7216) ${ }^{2}$

${ }^{1}$ Instituto Nacional de Câncer. Praça Cruz Vermelha 23, Centro. 20230-130 Rio de Janeiro RJ Brasil.

mario.silva@inca.gov.br

${ }^{2}$ Escola Nacional de Saúde Pública, Fundação Oswaldo Cruz. Rio de Janeiro RJ Brasil.

\begin{abstract}
This study was based on Giddens' Structuration Theory and aimed to analyze strategies adopted to ensure the rights of people with cancer in five Brazilian municipalities, anchored on the experience of managers and health professionals within the Brazilian Unified Health System (SUS). A multiple-case study design was conducted. The municipality was the unit of anal$y$ sis and oncology care-certified facilities were established as analytical sub-units. We analyzed the strategic behavior of ten managers and 15 health professionals from interviews. The results were systematized according to care, legal and social dimensions. The relevant elements expressed were the need to expand and organize the diagnostic and therapeutic cancer care network; the concern with the large number of lawsuits, both in light of economic sustainability of the health system and because of the promotion of inequalities; and the low resoluteness of social benefits, as they do not meet all people's needs. The adopted measures are considered insufficient to ensure the rights of people with cancer in the Brazilian Unified Health System.
\end{abstract}

Key words Patient rights, Neoplasms, Case reports, Health care, Unified Health System
Resumo Este estudo, fundamentado na Teoria da Estruturação de Giddens, buscou analisar as estratégias adotadas para garantir os direitos das pessoas com câncer em cinco municípios brasileiros, a partir da experiência de gestores e profissionais de saúde no contexto do Sistema Único de Saúde. Foi conduzido um estudo de casos múltiplos. A unidade de análise foi o município e a subunidade foi a unidade habilitada de atenção oncológica. Foi realizada a análise de conduta estratégica de dez gestores e 15 profissionais de saúde, a partir de entrevistas. Os resultados foram sistematizados em três dimensões: assistencial, judicial e social. Destacaram-se: a necessidade de expansão e organização da rede assistencial diagnóstica e terapêutica; a preocupação com o grande número de demandas judiciais, tanto pela sustentabilidade econômica do sistema de saúde, quanto pela promoção de iniquidades; a pouca resolutividade dos benefícios sociais, uma vez que não atendem a todos aqueles que necessitam. A percepção é de que as medidas adotadas são insuficientes para garantir os direitos da pessoa com câncer no sistema de saúde brasileiro.

Palavras-chave Direitos do paciente, Câncer, Estudo de casos, Assistência à saúde, Sistema Único de Saúde 


\section{Introdução}

O câncer tem se configurado como um importante problema de saúde pública, sendo previsto para 2030 a ocorrência global de, aproximadamente, 22 milhões de novos casos e de 13 milhões de mortes ${ }^{1}$. Em que pese sua relevância epidemiológica, observa-se um baixo investimento na formulação de políticas de prevenção e controle, e disparidades socioeconômicas que contribuem para grandes iniquidades no acesso aos sistemas de saúde, do diagnóstico ao tratamento ${ }^{2,3}$. Com o intuito de minimizar os impactos provocados pelas desigualdades, estratégias para garantir os direitos dos pacientes e sobreviventes do câncer vêm sendo debatidas ${ }^{4-6}$.

Existe uma variedade de documentos sobre direitos dos pacientes, mas apenas na Europa foi desenvolvida e publicada uma declaração de direitos das pessoas com câncer ${ }^{7,8}$. Esse documento está fundamentado em três princípios: i) o direito ao acesso à informação e ao envolvimento do paciente com o seu tratamento; ii) o direito ao acesso, em tempo oportuno, do diagnóstico ao tratamento; iii) o direito ao cuidado integral e de qualidade no sistema de saúde ${ }^{8}$. Segundo Jacobson et al. ${ }^{6}$, os países devem desenvolver suas declarações de direitos das pessoas com câncer.

O direito à saúde no Brasil é considerado um direito social consagrado pela Constituição Federal de 1988 e regulamentado pela Lei Orgânica da Saúde9. Em 2009, foi publicada a Carta de Direitos dos Usuários do Sistema Único de Saúde (SUS) com o intuito de garantir o acesso universal e igualitário dos cidadãos brasileiros às ações e serviços de saúde ${ }^{10}$. No intuito de garantir direitos à pessoa com câncer no SUS, três normativas podem ser destacadas: a Política Nacional para Prevenção e Controle do Câncer (PNPCC) ${ }^{11}$, a Lei $n^{\circ} 12.732 / 2012$, que estabelece o prazo de 60 dias para que o paciente oncológico diagnosticado receba o seu primeiro tratamento ${ }^{12} \mathrm{e}$ a Lei $\mathrm{n}^{\circ}$ $13.896 / 2019$, que define um prazo de 30 dias para que sejam realizados exames confirmatórios nos indivíduos que apresentarem neoplasia maligna como principal suspeita diagnóstica ${ }^{13}$. Além disso, são garantidos às pessoas com câncer alguns direitos previdenciários, tributários, financeiros, administrativos e judiciais, que buscam minimizar as vulnerabilidades acarretadas pelo adoecimento?.

A existência de normativas específicas para pacientes oncológicos constitui-se importante ferramenta de garantia ao direito constitucional à saúde e pode funcionar como uma estratégia de controle social ( $a d v o c a c y)^{3}$. Por outro lado, o não atendimento aos dispositivos legais pode se constituir em instrumento para judicialização, ampliando as iniquidades na atenção oncológica ${ }^{14}$.

Este estudo objetivou analisar as estratégias adotadas para garantir os direitos das pessoas com câncer em municípios brasileiros, a partir da experiência de gestores e profissionais de saúde no contexto do SUS, tendo a quimioterapia/ hormonioterapia para o tratamento do câncer de mama como condição marcadora.

\section{Método}

Trata-se de um estudo qualitativo fundamentado na Teoria da Estruturação de Giddens, que reconceitua a possível oposição existente entre o objetivismo e o subjetivismo, presente em determinadas teorias sociais, em "dualidade da estrutura" ${ }^{15}$. De acordo com O’Dwyer ${ }^{16}$, a sistematização dos elementos conceituais que compõem a teoria permite o emprego desta abordagem para a análise das práticas do SUS, subsidiadas por normas e regulamentos, e que são contextualizados por circunstâncias da ação. Considerando a dualidade da estrutura, as políticas podem ser analisadas quanto: i) à estrutura disponível, com seu implícito caráter limitador ou facilitador, relativo à formulação, correspondendo a uma "análise institucional"; ii), ao discurso e à ação do agente, relacionados à implantação, equivalendo a uma "análise de conduta estratégica"16.

Neste estudo foi realizada a análise de conduta estratégica ${ }^{15}$ dos gestores e dos profissionais de saúde. Embora a investigação tenha enfatizado as práticas, foram considerados os elementos da estrutura, especialmente as normativas relacionadas aos direitos da pessoa com câncer. Apoiado nesse referencial, foi conduzido um estudo de casos múltiplos do tipo integrado ${ }^{17}$. A unidade de análise principal (caso) foi o município e como subunidade de análise foi eleita a unidade habilitada de atenção oncológica.

Para selecionar os casos, considerou-se todos os municípios que tinham serviços de quimioterapia para tratamento de pacientes com câncer de mama. Os casos foram caracterizados quanto à macrorregião geográfica e ao tipo de região de saúde (RS) em que estavam inseridos (grupos 1 ao 5$)^{18}$. A tipologia utilizada se fundamenta na análise da situação socioeconômica e da oferta e complexidade dos serviços de saúde na região, reunindo municípios com características comuns e reduzindo o número de casos sem comprome- 
ter a variedade de informações analisadas. Foram selecionados cinco casos, sendo um município de cada tipo de RS diferente, situados em macrorregiões geográficas distintas.

Cada município incluiu uma unidade habilitada de atenção oncológica. Nos casos em que havia mais de um serviço, foram selecionados os estabelecimentos que realizavam maior número de procedimentos de quimioterapia, segundo o Sistema de Informações Ambulatoriais do SUS (SIA-SUS).

Os roteiros de entrevista empregados no estudo foram elaborados com o intuito de apreender as subjetividades e de conhecer a experiência dos sujeitos. Em cada município foram entrevistados o representante da gestão municipal da rede de atenção em oncologia, o gestor da assistência farmacêutica municipal e os profissionais da equipe mínima de terapia antineoplásica (médico, farmacêutico e enfermeiro) da unidade habilitada selecionada. No total, foram realizadas 25 entrevistas com agentes que estavam em posição estratégica para fornecer informações fundamentais para compreensão do fenômeno investigado.

Para evitar a identificação dos sujeitos, foram utilizadas siglas de correspondência com a função (GR: representante da gestão municipal da rede de atenção; GAF: gestor da assistência farmacêutica; MED: médico; FARM: farmacêutico; ENF: enfermeiro), seguidas do número correspondente ao tipo de RS onde o município estava inserido.

A análise ocorreu em três fases. O material foi lido e organizado para identificar os elementos mais frequentes, as similaridades e as divergências. A seguir, buscou-se reconhecer os núcleos de sentido, identificando os componentes de análise mais representativos dos temas destacados, por meio do uso do software QSR NVivo $11^{\circledR}$. Os resultados foram sistematizados em três dimensões: assistencial, judicial e social. Por fim houve interpretação de resultados e inferência analítica, com a finalidade de dar significado e validade aos achados.

A pesquisa obedeceu aos pressupostos da Resolução do Conselho Nacional de Saúde $n^{\circ}$ $466 / 2012$, que regulamenta pesquisas envolvendo seres humanos. O estudo foi aprovado pelo Comitê de Ética em Pesquisa da Escola Nacional de Saúde Pública Sérgio Arouca, registrado sob no 55992716.8.0000.5240.

\section{Resultados e discussão}

As três dimensões de análise que emergiram da pesquisa estiveram centradas, especialmente, em dois princípios fundamentais do direito à pessoa com câncer, um relacionado ao acesso e outro relativo ao cuidado integral e de qualidade no sistema de saúde $e^{7,8,11}$. Esses aspectos ganham importantes contornos na análise da conduta estratégica, uma vez que na Teoria da Estruturação as práticas são socialmente construídas, porém pautadas por estratégias de controle definidas por limites contextuais ${ }^{15}$.

\section{Dimensão Assistencial}

Nessa dimensão, um dos pontos mais destacados foi a necessidade da expansão da rede, como importante estratégia para ampliação do cuidado em oncologia, com impacto coletivo e social. A restrição de cenários de atendimento aos pacientes oncológicos pode resultar em diminuição importante na sobrevida e na qualidade de vida dos sobreviventes do câncer ${ }^{2}$. Dificuldades relacionadas aos diversos serviços necessários ao controle do câncer têm sido apontadas na literatura, tais como: i) a distância a ser percorrida até um centro de tratamento oncológico, significando uma grande barreira de acesso ${ }^{19}$; ii) o longo tempo de espera para se realizar o diagnóstico da doença, devido à sobrecarga do limitado número de laboratórios de anatomopatologia e histopatologia ${ }^{20}$; iii) a escassez de profissionais capacitados para atuar na atenção ao câncer, nos diversos níveis de atenção ${ }^{21}$. Suplantar essas e outras dificuldades para expandir a rede é um desafio necessário:

Mas a gente tem dado uma resposta legal com a expansão da rede da atenção básica. Pelo menos esses diagnósticos tão sendo cada vez maiores e mais precoces. [...] Por outro lado, preocupa, porque ... não adianta só o diagnóstico. Você cria expectativa e não dá continuidade. Então tem que vir junto, diagnóstico e tratamento e acompanhamento posterior (GR4).

Apesar da expansão apontada pelo entrevistado, a preocupação apresentada é legítima. Para se garantir o direito da pessoa com câncer é fundamental que se estruture o todo e não apenas uma parte da rede de atenção ${ }^{11,18}$. De acordo com a Teoria da Estruturação dois processos estariam envolvidos na realização da ação: a racionalização e a motivação ${ }^{15}$. O primeiro se refere à compreensão teórica dos atores (que subsidia a ação) e o segundo ao potencial para a ação, que não se vincula à sua continuidade ${ }^{16}$. 
Outro ponto destacado se refere a uma iniciativa institucional, realizada no município 3 , que inclui a divulgação de ações de prevenção, a realização de diagnóstico precoce e a disseminação de informações sobre o fluxo de atendimento institucional:

Tem a [iniciativa institucional] [...] que vai a equipe multiprofissional, [...] a gente pega um município, [...] entra em contato com a Secretaria de Saúde, [...] aí a gente vai dar palestras no auditório para essa comunidade, [sobre] prevenção de câncer [...]. Inclusive, a partir desse projeto, a gente teve alguns pacientes que a gente conseguiu trazer para vir para o centro de oncologia para tratamento... (ENF3).

A realização desse projeto demonstra o quanto a unidade habilitada reconhece o seu papel na rede de atenção ${ }^{11}$, não sendo apenas uma executora do tratamento. Cumpre com o que foi contratualizado mas amplia as suas ações para fortalecimento da linha de cuidado em oncologia. De acordo com Giddens ${ }^{15}$, o agente é capaz de fazer a diferença. Presume-se que uma ação, que se utiliza de recursos para se concretizar, é dotada de poder, no sentido de capacidade de mudança. No âmbito do direito da pessoa com câncer, esse tipo de estratégia é fundamental. $\mathrm{O}$ acesso à informação possibilita ao indivíduo compreender a necessidade de adotar medidas de prevenção e/ ou de se envolver com o seu tratamento ${ }^{6-8,11}$.

Uma iniciativa, também destacada, foi o " $\mathrm{de}$ livery da hormonioterapia" para pacientes que residem em outros municípios. Utilizar as farmácias das unidades básicas de saúde para dar suporte para os centros de oncologia parece ser uma estratégia importante para continuidade do cuidado ao paciente com câncer e no processo de estruturação da rede de atenção em oncologia, principalmente por minimizar possíveis barreiras de acesso geográficos ${ }^{21}$ :

A gente tem municípios próximos e a gente separa as medicações. [...] a gente separa todas as pacientes que são dessa região, a gente põe o nome em cada caixinha [...] a gente coloca no container e a prefeitura busca a medicação. Então a prefeitura pega essa medicação, leva pra farmácia, e o paciente de lá busca na própria instituição (FARM1).

Do ponto de vista do acesso ao tratamento, um aspecto enseja reflexão: a responsabilidade do tratamento deve ser compartilhada ${ }^{11}$, não apenas a provisão dos medicamentos. Não é possível saber se os pacientes estão utilizando os medicamentos de forma apropriada ou apresentando efeito adverso que precise ser manejado adequadamente. Não há seguimento "remoto" dos pacientes atendidos no município A pelo município $\mathrm{B}$, ou conhecimento sobre a forma com que o município A faria o seguimento dos pacientes atendidos pelo município vizinho; não foi relatada esta necessária parte do acordo entre os entes. O farmacêutico, do município 4, mostra essa inquietude:

... é muita cidade do interior, a maioria dos pacientes é do interior. Às vezes, vem motorista de ambulância pegar, eu não tenho contato com o paciente [...] vem pegar remédio pra dez pacientes. Então que garantia que eu vou te afirmar [...] que esse remédio tá sendo entregue para o paciente? Que o paciente tá tomando direito? Eu não sei. Eu já fiquei sabendo de caso que o paciente estava com três caixas em casa, não queria tomar (FARM4).

A fala da entrevistada se aproxima do que Giddens ${ }^{15}$ denomina de monitoramento reflexivo. Trata-se de uma característica crônica da atividade rotineira e envolve a conduta do agente, e dos demais. Os atores controlam e regulam as suas atividades, frente ao contexto e esperam que os outros façam o mesmo, por conta própria ${ }^{16}$. Na perspectiva dos direitos da pessoa com câncer, é fundamental que esse tipo de atividade ocorra de forma estruturada, uma vez que não se pode negar o risco de desvio da hormonioterapia encaminhada, considerando que a entrega não foi realizada diretamente ao paciente:

Já teve caso de paciente vir aqui, falar: "ó, eu vim buscar meu remédio". [E eu responder:] "Não, o seu remédio já saiu" (FARM4).

A 'terceirização informal dos serviços' foi uma outra estratégia enunciada pelos profissionais do município 2 . O relato mostra o que pode ser considerado como "desvio de processo", a partir da significação institucional ${ }^{15}$. Segundo os entrevistados, quando faltam medicamentos para realizar o tratamento quimioterápico, os pacientes são encaminhados para um serviço da iniciativa privada, não contratualizado pelo SUS, para a realização do procedimento. A unidade pública habilitada preenche a Autorização de Procedimento de Alta Complexidade (APAC) e o valor ressarcido é cambiado para o serviço privado:

O nosso serviço ele tem um... contrato com uma clínica particular, que também fornece serviço de quimioterapia. Quando falta medicação no estado, o paciente é referenciado pra esse serviço privado. [...] Há época em que, [...] 60\% dos nossos pacientes estão lá. E há época que $90 \%$ dos nossos pacientes estão aqui. Então é muito sazonal, por culpa do processo de aquisição, da medicação... (FARM2).

Embora se compreenda a intenção de evitar 
o risco de descontinuidade do tratamento, frente ao desabastecimento de medicamentos, é importante ponderar o atendimento realizado em um serviço não habilitado pelo SUS. Não é possível saber se as mesmas condições estão sendo atendidas. Ademais, a desorganização do sistema traz situações conflituosas para os pacientes, atendidos por profissionais diferentes, em estruturas físicas e operacionais distintas ${ }^{18}$. Do ponto de vista prático, é um plano de contingência ineficiente, uma vez que não promove soluções definitivas ao problema identificado - a falta de medicamentos - e favorece o uso inadequado dos recursos públicos.

\section{Dimensão Judicial}

A judicialização aparece, pela concepção dos entrevistados, como uma consequência esperada da desorganização da rede e da falta de acesso, sendo entendida como uma forma de garantir os direitos da pessoa com câncer. Essa dimensão foi abordada por todos os sujeitos. Na perspectiva dos gestores, três apontam aspectos que se destacam:

i) produção de iniquidade pela realidade do sistema paralelo ${ }^{22}$ :

... eu sempre digo que eu sou contra a judicialização [...]. Ela não faz parte do SUS. É um sistema paralelo. [...] e você não sabe o resultado disso, como é que funciona e pra quem que funciona (GR4).

ii) escolhas trágicas entre atender as necessidades individuais frente as coletivas ${ }^{23}$ :

... aparecem esses [processos] judiciais...Às vezes, por exemplo, a gente tá com recurso para fazer... cirurgia de catarata de 300 pessoas. [...] Ficam 300 pacientes esperando a cirurgia de catarata, enquanto tem um paciente recebendo tratamento oncológico que não tem um custo-efetividade aprovado (GAF1).

iii) judicialização como estratégia mercado$\operatorname{lógica}^{14}$ :

... quando não se faz uma politica com mais consistência e que se antecipe a algumas situações, a gente fica à mercê do mercado e das ações de judicialização (GAF3).

As preocupações são claras: o dispendioso investimento, que paradoxalmente reflete em desassistência de diversos usuários do $\mathrm{SUS}^{23}$, e a não integralidade do cuidado à pessoa com cân$\operatorname{cer}^{11,24}$.

Outro aspecto relevante se refere a importância da aplicabilidade integral e constante dos dispositivos normativos existentes ${ }^{10-13}$, com intuito de se garantir os direitos da pessoa com câncer, demandando mudanças que possam minimizar o número de demandas judiciais. Considerando o contexto da judicialização, no qual prevalecem a regulação normativa e o princípio da legitimação, o que Giddens ${ }^{15}$ denomina de instituições legais, é fundamental que as regras sejam adequadamente estabelecidas, evitando possíveis distorções.

Do ponto de vista dos profissionais, as falas estiveram mais centradas nas motivações ${ }^{15}$, e surgem a partir da prescrição médica (na perspectiva clínica), e que podem constituir-se força motriz da judicialização. O médico vê sua "responsabilidade" profissional como quase antagônica frente à sua atuação no sistema:

Normalmente, a gente como médico, tem que esclarecer para o paciente quais são as possibilidades que existem na comunidade científica, é nossa obrigação. A gente não pode omitir, assim, banir o paciente de ter o benefício de uma medicação porque o SUS não cobre, né? [...] A partir daí o paciente, se tiver interesse, eles entram via judicial para conseguir a medicação. O que vem se tornando uma coisa muito frequente no SUS, no Brasil inteiro... (MED2).

As questões destacadas apontam para a atualização científica, que na área da oncologia é bastante dinâmica e significativa ${ }^{6}$. Em 2018, havia mais de 1.000 pesquisas clínicas em andamento somente com fármacos antitumorais ${ }^{25}$. É praticamente impossível para qualquer sistema de saúde do mundo manter todas as novas terapias acessíveis para a população, devido a insuficiência de informações sobre efetividade e segurança dos novos medicamentos, como também pelo alto custo das novas tecnologias.

Não obstante, a decisão médica é sempre, ou quase sempre, o iniciador da demanda judicial de medicamentos. Como a prescrição é aceita como soberana pela Justiça ${ }^{22}$, a necessidade real do paciente pode não ser questionada, especialmente no câncer, face às questões psicossociais subjacentes, à complexidade e à urgência da atenção.

Os profissionais apontam, ainda, as distorções que podem advir do trâmite judicial, do processo em si, relativos ao início do tratamento:

[As demandas judiciais] promovem atrasos, porque a gente fornece o laudo pro paciente explicando qual o remédio, qual a indicação, porque ele deve tomar, e qual o estudo que a gente referenciou nisso. Então ele vai no Ministério Público ou na Defensoria Pública, dá entrada no processo. Aí eles reconhecem, pegam o laudo, vêm aqui na secretaria. Pedem uma notificação da secretaria se eles 
realmente não têm esse remédio, para depois dar entrada no processo. Isso leva no mínimo [...] 30 dias. Então assim, para o paciente começar a usar a medicação vai ser 60 dias... (MED2).

E à continuidade do cuidado:

A gente processa o estado. [...] O estado vem trazendo vários empecilhos, fica pegando detalhe de relatório, ou às vezes até libera, mas aí o procurador vem com uma liminar e suspende o tratamento do paciente. Então, o paciente começa e daqui a pouco para o tratamento na metade... (MED5).

Atraso e descontinuidade dos tratamentos são condições preocupantes, pois podem promover o agravamento dos casos, aumentar a chance de recidiva em situações já controladas e elevar o risco de mortalidade ${ }^{19}$. De acordo com a análise de conduta estratégica, o uso do poder em sistemas sociais, nesse caso, a judicialização, pressupõe relações reguladas de autonomia e dependência entre diferentes atores em um cenário de interação social ${ }^{15}$. Nesse sentido, apesar de ser uma prática recorrente e intencionada a suplantar barreiras, os profissionais compreendem que o trâmite judicial não é o caminho mais adequado para que o paciente exerça o seu direito de acesso ao tratamento.

Duas outras situações foram apontadas. A primeira se refere à instituição abonando a conduta médica e adotando medidas para que os pacientes acionem a justiça:

Aqui é assim, o hospital me dá total autonomia para eu solicitar o que eu quiser para o paciente meu. [...] A gente dispõe de um advogado que não é contratado do hospital, ele vem aqui de forma voluntária e ele faz ações para os pacientes de SUS (MED5).

As relações duvidosas estabelecidas na impetração de demandas judiciais em oncologia vêm sendo analisadas em outros estudos, que elucidam interesses particulares em detrimento dos coletivos $^{24,26,27}$. Institucionalizar esse tipo de prática pode ampliar iniquidades, propiciando maiores dificuldades para o sistema responder adequadamente às necessidades de saúde da população.

A segunda é que, em havendo parecer favorável pelo Ministério Público para a ação judicial impetrada, os pacientes recebem, dos entes federados, o valor financeiro para a compra dos medicamentos, transferindo para o paciente a responsabilidade da aquisição do produto:

Ele entra na justiça, ele ganha, como o estado não consegue comprar, o estado deposita o dinheiro na conta desse paciente. O paciente que tem que efetuar a compra. O paciente mal conhece a me- dicação, sabe no papel que tá escrito $1 \mathrm{mg}$, mas ele não entende; ele não tem acesso ao sistema de compra, ele não sabe de quem comprar (FARM2).

$O$ paciente não tem conhecimento técnico e nem administrativo suficiente para realizar a aquisição, pois existem questões de ordem sanitária, relacionadas ao transporte, ao armazenamento, às características do produto e à falsificação, dentre outras, que precisam ser observadas no momento da compra de um medicamento ${ }^{28}$. Por outro lado, mesmo que o paciente tenha conseguido adquirir, onde será preparado para infusão ou dose? Riscos importantes são agregados ao preparo de um produto sem garantia de que todos os requisitos de boas práticas foram rigorosamente cumpridos, configurando atitude perigosa e desprovida de responsabilidade sanitária.

Três municípios ( 1,3 e 5 ) relataram experiências exitosas relacionadas à resolução de conflitos por meio de estratégias extrajudiciais:

Nós temos um grupo de trabalho com um juiz federal, juiz estadual, pessoal do estado, tem o pessoal da Advocacia da União, Defensoria Pública, todos os hospitais ..., filantrópico, particular ou SUS... E a gente tem feito reuniões mensais para tentar verificar fluxos de encaminhamentos, trabathar de que forma a gente pode fazer um trabalho melhor, envolvendo juizes, envolvendo secretaria de saúde e secretaria de estado (GAF5).

A estratégia destacada aponta para a construção de consensos e de exercício de $a d v o c a c y^{3}$, com o intuito de garantir o acesso, a continuidade e qualidade do cuidado prestado ao indivíduo com câncer ${ }^{11}$, com dinâmica de integração social que se estabelece a partir das interações entre os diversos atores envolvidos promovendo mudanças significativas nos sistemas ${ }^{15}$. Nessas experiências predominam ênfase na negociação, pactuação e concessão; se estabelecem estratégias de implementação de políticas públicas mais consistentes; incorporase a perspectiva da sociedade civil e da instituição na construção dos consensos e interpretação dos direitos constitucionais; e se consideram os diferentes contextos em que as demandas estão inseridas para adoção de medidas mais efetivas para sua resolução ${ }^{29}$. Pela sua dinâmica, pluralidade e intencionalidade é importante estratégia para se garantir o direito da pessoa com câncer.

\section{Dimensão social}

A garantia da saúde, como um direito constitucional reconhecido, alberga um conjunto de benefícios sociais que podem ser requeridos pelos cidadãos em situações de doençą ${ }^{10}$. No caso 
do tratamento oncológico, a Lei no 12.732/2012 estabelece, em seu parágrafo primeiro, que o paciente com câncer receberá, gratuitamente, no SUS, todos os tratamentos necessários ${ }^{12}$. Um dos pontos mais destacados pelos entrevistados foi o auxílio social, que é parte de um conjunto de direitos dos pacientes, inclusive para aquisição de medicamentos:

... às vezes o paciente, ele fala que não tem condições socioeconômicas de adquirir o produto. Ai nós temos o serviço social que faz uma avaliação do paciente. [...] Aí ele encaminha para a nossa farmácia municipal. Daí nós temos um contrato com a farmácia, que ele adquire o medicamento e fornece para o paciente. Enquanto perdurar a sintomatologia (GAF5).

Muito embora seja possível perceber na fala do agente que a ação envolve processos de racionalização e motivação ${ }^{15}$, a estratégia é pouco resolutiva. $\mathrm{O}$ atendimento individualizado, embora importante, fere um princípio constitucional e promove iniquidades, pois apenas usuários que conhecem seus direitos conseguem ter acesso ao tratamento?.

O “pagamento administrativo", também foi ressaltado como uma das iniciativas adotadas pelos municípios, mas estaria condicionado à capacidade de alguns em assumir financeiramente o custo de tratamento não previsto pelas diretrizes publicadas pelo Ministério da Saúde:

[...] nós pedimos que o médico solicitante faça uma justificativa técnica e que ele cite pelo menos três evidências para a gente, enquanto técnico aqui na secretaria de saúde, [...] poder fazer a liberação ou não desse pagamento administrativo (GR5).

Uma vez que não foram avaliadas, protocoladas e/ou incorporadas pela Comissão Nacional de Incorporação de Tecnologias do SUS (Conitec), essas terapias podem trazer riscos aos pacientes. A adoção desta iniciativa, como resposta governamental, além de favorecer iniquidades em um sistema que deveria ser único e universal, é frágil do ponto de vista político, pois é dependente do poder exercido pelo agente ${ }^{15}$. Ou seja, caso ocorra mudança na gestão a medida poderá ser descontinuada.

Qualquer iniciativa que busque resolver conflitos contornando as regras vigentes não traz sustentabilidade ao SUS e não garante a integralidade do cuidado. Gastos judiciários, extrajudiciários, administrativos ou por benefício de assistência à saúde apenas criam mecanismos alternativos, resultando ainda em pressão para a incorporação de tecnologias ${ }^{30,31}$.

$\mathrm{O}$ apelo social do câncer favorece a mobilização das pessoas, individual ou coletivamente, sendo um importante suporte para as instituições de saúde, em especial para os centros de tratamento de câncer ${ }^{32,33}$, buscando suprir algumas necessidades não atendidas pelos órgãos governamentais. Além do apoio social, por vezes realizam suporte espiritual e terapêutico ${ }^{32}$, por meio das Organizações Não Governamentais (ONG):

[...] tem a casa de apoio [da ONG], onde elas acolhem alguns pacientes do interior, principalmente, quem não tem onde ficar e que precisam fazer, por exemplo, um tratamento de radioterapia, que são vários dias seguidos, ou uma quimioterapia que são vários dias seguidos (FARM3).

E da ação do voluntariado:

As pacientes, quando elas passam por todo o tratamento, elas têm o acompanhamento de um grupo de apoio de mulheres [voluntárias] do serviço de oncologia... (FARM2).

Porém, é fundamental que não se transfira para as entidades de apoio o papel institucional legitimado ${ }^{15}$, que deve ser exercido pelos entes federados e unidades habilitadas ${ }^{11}$.

O Tratamento Fora do Domicílio (TFD) é um benefício social que oferece auxílio ao paciente atendido na rede pública e/ou conveniada de saúde pelo SUS, em outro município ou Unidade da Federação, com distância mínima de $50 \mathrm{~km}$ da sua residência ${ }^{9,34}$. Embora, seja uma importante estratégia para diminuir as barreiras de acesso geográfico, os entrevistados expressaram preocupação com a eficiência do TFD, quanto ao início do tratamento:

A gente pede o TFD e passa tudo via secretaria. Eles que entram em contato com o serviço solicitando a vaga e daí eles que programam a viagem do paciente [...] às vezes esse processo demora 60 dias. [...] Porque assim, depende de ter vagas em outros serviços, e esse serviço disponibilizar a vaga... (MED2).

À sua continuidade:

[...] nós temos $60 \%$ de pacientes de fora, [...] tem paciente que às vezes chega às 7 horas da manhã e sai 6 horas da tarde, [...] fica esperando o carro da prefeitura e é cansativo. Ainda mais para a mulher que ainda tem família, tem casa. Então assim, é complicado (FARM1).

E com relação ao uso dos recursos públicos:

Quanto gasto tem cada ambulância pra trazer paciente pra cá? Passar o dia inteiro, aí tem comi$d a$, ai às vezes tem repouso, porque o paciente tem que ficar a semana inteira aqui. Aí tem que arrumar casa de apoio, ou o próprio paciente tem que custear o custo dele no hotel. Então isso tudo gera muito custo... (ENF4).

$\mathrm{O}$ atraso no início do tratamento pode implicar no agravamento do câncer ${ }^{6}$, e os riscos de 
descontinuidade promovem desfechos negati$\operatorname{vos}^{19}$. Os recursos financeiros investidos no TFD funcionam como facilitadores da mobilidade de acesso $^{34}$, dando suporte ao sistema logístico aos usuários com câncer, quando necessário ${ }^{11}$. No entanto, a disponibilidade de recursos para TFD ${ }^{9}$ não implica necessariamente no seu uso, uma vez que questões sociais e clínicas podem inviabilizar o deslocamento do paciente para outras localidades:

... a nossa dificuldade de acesso, que a maioria é aéreo, nem todos os pacientes conseguem subir num avião. Pacientes que têm metástase cerebral não conseguem andar, pra sentar no avião, fazer uma viagem de uma hora e descer pra radio[terapia], entende? E se for de ônibus, aqui é pior ainda, porque é no mínimo 10 horas. Então, pacientes de [câncer de] pulmão que precisam de oxigênio não conseguem sair daqui pra fazer radioterapia. Paciente que tem compressão medular, que não deambula [...], é difícil você conseguir mobilizar eles com o transporte aéreo, porque eles não conseguem sentar muito tempo... é cansativo, têm dor, pra poder fazer a radioterapia (MED2).

Considerando as experiências dos entrevistados, o uso do TFD como garantia de direito à pessoa com câncer parece se traduzir como um recurso limitado de ação, pois apesar de previsto em normativa seu emprego prático é complexo, sendo essa, portanto, uma dualidade da estrutu$\mathrm{ra}^{15}$.

Como limitações do estudo, apontam-se a impossibilidade de entrevistar maior número de profissionais e de inclusão de outras unidades habilitadas de atenção oncológica nos casos selecionados. Porém, acredita-se que os indivíduos que participaram das entrevistas conseguiram ter uma abordagem sistêmica sobre a temática investigada.

\section{Considerações finais}

Ao se analisar as estratégias adotadas para garantir os direitos dos pacientes com câncer no contexto do SUS, identificou-se um conjunto de experiências que vêm sendo vivenciadas pelos gestores e profissionais de saúde, com diferentes perspectivas. Foi possível notar, nas falas dos entrevistados, a existência de explicações sobre o quê e o porquê faziam determinadas ações, o que Gidden ${ }^{14}$ denomina de cognoscitividade - um mecanismo de defesa do sujeito, uma forma de significação, um sistema de interpretação, uma tentativa de dar sentido às atitudes adotadas.

De acordo com os entrevistados, a expansão e a organização da rede assistencial, diagnóstica e terapêutica, são estratégias que devem ser empreendidas para que os pacientes usufruam adequadamente dos seus direitos. A temática da judicialização ganhou grande destaque na pesquisa. Foi demonstrada intensa preocupação com a sustentabilidade econômica do sistema de saúde, mediante o volume significativo de demandas judiciais e com suas consequências, com destaque para os desfechos clínicos desfavoráveis e o prejuízo assistencial para outras condições de saúde, promovendo iniquidades. Ressaltou-se, ainda, a pouca resolutividade dos benefícios sociais, uma vez que não atendem a todos aqueles que necessitam recebê-los.

Os temas abordados revelaram a desarticulação existente no sistema de saúde público brasileiro. Em oncologia o alcance de resultados positivos é dependente da integração de uma rede de cuidados especializados (média e alta complexidade) e não especializados (cuidados primários, cuidados paliativos, saúde mental e outros). É preciso que os diversos agentes, envolvidos no sistema, compreendam as influências que afetam as circunstâncias da ação e promovam a transformação das práticas realizadas, possibilitando a integração das ações e serviços de saúde, para além das regras e recursos disponíveis, e assim consigam garantir os direitos da pessoa com câncer. 


\section{Colaboradores}

Os autores contribuíram igualmente no delineamento do estudo, análise e interpretação dos dados, redação e revisão crítica do artigo, e aprovaram a versão final encaminhada.

\section{Referências}

1. Fidler MM, Bray F, Soerjomataram I. The global cancer burden and human development: A review. Scand J Public Health 2018; 46(1):27-36.

2. Prager GW, Braga S, Bystricky B, Qvortrup C, Criscitiello C, Esin E, Sonke GS, Martínez GA, Frenel JS, Karamouzis M, Strijbos M, Yazici O, Bossi P, Banerjee S, Troiani T, Eniu A, Ciardiello F, Tabernero J, Zielinski CC, Casali PG, Cardoso F, Douillard JY, Jezdic S, McGregor K, Bricalli G, Vyas M, Ilbawi A. Global cancer control: responding to the growing burden, rising costs and inequalities in access. ESMO Open 2018; 3(2):e000285.

3. McCanney J, Johnson T, Bandini LA, Chambers S, Bonar L, Carlson RW. Advocating for Equity in Cancer Care. J Natl Compr Canc Netw2019; 17(9):1043-1048.

4. Spinks T, Albright HW, Feeley TW, Walters R, Burke TW, Aloia T, Bruera E, Buzdar A, Foxhall L, Hui D, Summers B, Rodriguez A, DuBois R, Shine KI. Ensuring quality cancer care: a follow up review of the Institute of Medicine's 10 recommendations for improving the quality of cancer care in America. Cancer 2012; 118(10):2571-2582.

5. Schear RM, Manasco L, McGoldrick D, Kajana K, Rosenthal L, McMikelA, Lins N. International framework for cancer patient advocacy: empowering organizations and patients to create a national call to action on cancer. J Glob Oncol 2015; 1(2):83-91.

6. Jacobson JO, Berry LL, Spears PA, Steffensen KD, Attai DJ. Proposing a Bill of Rights for Patients with Cancer. J Clin Oncol 2020; 16(3):121-123.

7. Lawler M, Le Chevalier T, Banks I, Conte P, De Lorenzo F, Meunier F, Pinedo HM, Selby P, Murphy MJ, Johnston PG. A Bill of Rights for patients with cancer in Europe. Lancet Oncol 2014; 15(3):258-260.

8. Lawler M, Banks I, Law K, Albreht T, Armand JP, Barbacid M,Barzach M, Bergh J, Cameron D, Conte P, Braud F, Gramont A, Lorenzo FD, Diehl V, Diler S, Erdem S, Geissler J, Gore-Booth J, Henning G, Højgaard L, Horgan D, Jassem J, Johnson P, Kaasa S, Kapitein P, Karjalainen S, Kelly J, Kienesberger A, Vecchia CL, Lacombe D, Lindahl T, Löwenberg B, Luzzatto L, Malby R, Mastris K, Meunier F, Murphy M, Naredi P, Nurse P, Oliver K, Pearce J, Pelouchov J, Piccart M, Pinedo B, Spurrier-Bernand G, Sullivan R, Tabernero Jm Velde CV, Herk BV, Vedsted P, Waldmann A, Weller D, Wilking N, Wilson R, Yared W, Zielinski C, Hausen HZ, Chevalier TL, Jonston P, Selby P. The European cancer patient's bill of rights, update and implementation 2016. ESMO Open 2016; 1(6):e000127.

9. Campos JRIS. Direito fundamental à saúde: uma análise da proteção jurídica às pessoas acometidas por neoplasia maligna. Rev Videre 2015; 7(13):34-48.

10. Brasil. Ministério da Saúde (MS). Carta dos direitos dos usuários da saúde. $3^{\mathrm{a}}$ ed. Brasília: MS; 2011.

11. Brasil. Ministério da Saúde (MS). Anexo IX da Portaria de Consolidação $\mathrm{n}^{\circ} 2$, de 28 de setembro de 2017. Dispõe sobre a Política Nacional para Prevenção e Controle do Câncer (PNPCC). Diário Oficial da União 2017; 3 out.

12. Brasil. Lei ${ }^{\circ} 12.732$, de 22 de novembro de 2012. Dispõe sobre o primeiro tratamento de paciente com neoplasia maligna comprovada e estabelece prazo para seu início. Diário Oficial da União 2012; 23 nov. 
13. Brasil. Lei no 13.896 , de 30 de outubro de 2019. Altera a Lei $n^{\circ} 12.732$, de 22 de novembro de 2012, para que os exames relacionados ao diagnóstico de neoplasia maligna sejam realizados no prazo de 30 (trinta) dias, no caso em que especifica. Diário Oficial da União 2019; 31 out.

14. Vidal TJ, Moraes EL, Retto MPF, Silva MJS. Demandas judiciais por medicamentos antineoplásicos: a ponta de um iceberg? Cien Saude Colet 2017; 22(8):25392548.

15. Giddens A. A constituição da sociedade. São Paulo: Martins Fontes; 2003.

16. O’Dwyer G. Estudos de políticas e a teoria da estruturação de Giddens. In: Baptista TWF, Azevedo CS, Machado CV, organizadores. Políticas, planejamento e gestão em saúde: abordagens e métodos de pesquisa. Rio de Janeiro: Editora Fiocruz; 2015. p. 173-192.

17. Yin RK. Estudo de caso: planejamento e métodos. Porto Alegre: Bookman; 2015.

18. Silva MJS, O’Dwyer G, Osorio-de-Castro CGS. Cancer care in Brazil: structure and geographical distribution. BMC Cancer 2019; 19(1):987.

19. Silva MJS, Melo ECP, Osorio-de-Castro CGS. Origindestination flows in chemotherapy for breast cancer in Brazil: implications for pharmaceutical services. Cien Saude Colet 2019; 24(3):1153-1164.

20. Fayer VA, Guerra MR, Cintra JRD, Bustamante-Teixeira MT. Sobrevida de dez anos e fatores prognósticos para o câncer de mama na região Sudeste do Brasil. Rev Bras Epidemiol 2016; 19(4):766-778.

21. Nekhlyudov L, O'Malley DM, Hudson SV. Integrating primary care providers in the care of cancer survivors: gaps in evidence and future opportunities. Lancet Oncol 2017; 18(1):e30-e38.

22. Pereira JG, Pepe VLE. Acesso a medicamentos por via judicial no Paraná: aplicação de um modelo metodológico para análise e monitoramento das demandas judiciais. Rev Dir Sanit 2015; 15(2):30-45.

23. Domingos LO, Rosa GFC. O direito fundamental e coletivo à saúde no contexto da judicialização. Cad Ibero-Amer Dir Sanit 2019; 8(2):82-99.

24. Deprá AS, Ribeiro CDM, Maksud I. Estratégias de instituições da sociedade civil no acesso a medicamentos para câncer de mama no SUS. Cad Saude Publica 2015; 31(7):1517-1527.

25. Beinse G, Tellier V, Charvet V, Deutsch E, Borget I, Massard C, Hollebecque A, Verlingue L. Prediction of drug approval after phase I clinical trials in oncology: RESOLVED2. JCO Clin Cancer Inform 2019; 1:1-10.

26. Silva HP, Pimenta KKP. A atuação de advogados e organizações não governamentais na judicialização da saúde pública no Brasil: a quem será que se destina? Cad Ibero-Amer Dir Sanit 2017; 6(1):207-227.
27. McCoy MS, Carniol M, Chockley K, Urwin JW, Emanuel EJ, Schmidt H. Conflicts of interest for patient-advocacy organizations. N Engl J Med 2017; 376(9):880-885.

28. Costa AA, Chaves GC, Brito MA. Procurement of medications in the Brazilian public sector: the search for quality in bidding processes. Rev Bras Farm Hosp Serv Saude 2019; 10(2):0413.

29. Asensi FD. Judicialização ou juridicização? As instituições jurídicas e suas estratégias na saúde. Physis 2010; 20(1):33-55.

30. Biehl J, Socal MP, Amon JJ. The judicialization of health and the quest for state accountability: evidence from 1,262 lawsuits for access to medicines in southern Brazil. Health Hum Rights 2016; 18(1):209-220.

31. Aragão SM, Silva ALC, Ditterich RG, Kusma SZ. Reclamatórias em saúde recebidas pelo Ministério Público do Estado do Paraná: um estudo do CAOP de Proteção à Saúde Pública. Rev Dir Sanit 2019; 20(1):223-243.

32. Lorhan S, Van-Der-Westhuizen M, Gossmann S. The role of volunteers at an outpatient cancer center: how do volunteers enhance the patient experience? Support Care Cancer 2015; 23(6):1597-1605.

33. Thrift-Perry M, Cabanes A, Cardoso F, Hunt KM, Cruz TA, Faircloth K. Global analysis of metastatic breast cancer policy gaps and advocacy efforts across the patient journey. Breast 2018; 41:93-106.

34. Brasil. Ministério da Saúde (MS). Portaria n ${ }^{\circ} 55$, de 24 de fevereiro de 1999. Dispõe sobre a rotina do Tratamento Fora de Domicílio no Sistema Único de Saúde - SUS, com inclusão dos procedimentos específicos na tabela de procedimentos do Sistema de Informações Ambulatoriais do SIA/SUS e dá outras providências. Diário Oficial da União 1999; 26 fev.

Artigo apresentado em 03/07/2020

Aprovado em 26/11/2020

Versão final apresentada em 28/11/2020

Editores-chefes: Romeu Gomes, Antônio Augusto Moura da Silva 